\title{
DIE GROSSEN ORATORIEN-PRODUKTIONEN DER TONKÜNSTLER-SOCIETÄT IN WIEN - KONTRAPUNKT ODER NACHFOLGER DER HÖFISCHEN ORATORIENPFLEGE DES BAROCK?
}

Wie auch die Oper war die Oratorienproduktion in Wien von ihren Anfängen unter Kaiserin Eleonora Gonzaga II. um 1640 eine vorwiegend höfische Gattung. Vor allem die Aufführungen der Fastenoratorien, die in Wien zur speziellen Form des Sepolcro modifiziert wurden, verliefen ab 1660 in streng geregelten Bahnen: „Jährlich wird am Gründonnertag in der Kapelle Eleonoras II. eines ihres Kapellmeisters gegeben - natürlich nur bis in ihr Todesjahr 1686 -, am Karfreitag dann ein weiteres vom kaiserlichen Maestro di cappella oder von seinem Stellvertreter in der Hofburgkapelle."1 Ab dem Tod Eleonoras wurde nur noch ein Sepolcro pro Fastenzeit aufgeführt, nach dem Tod Leopolds änderte sich der Charakter des Sepolcro solcherart, dass von einem Sepolcro im engeren Sinn nicht mehr gesprochen werden kann: Das Sepolcro verlor seine Charakteristika und näherte sich dem Oratorium $a^{2}$ - nur der Aufführungsort blieb der selbe: die Hofburgkapelle. Mit dem Tod Karls VI. „lässt das Interesse an Oratorien am Wiener Hof insgesamt sehr stark nach". ${ }^{3}$

1745 überrascht eine Anzeige im Wienerischen Diarium; sie kündigt mit 6. März 1745 eine Akademie an, die als Start der Wiener Fastenkonzerte gesehen werden kann. Sollte Johann Joseph Fürst Khevenhüller-Metsch mit der kurzen Anmerkung in seinem Tagebuch Recht haben, wäre der Erfolg (vorerst) eher mäBig gewesen: ,[...] Anheut hatte der Impressario deren Spectacles in dem Balhauß Concerti di musica zu geben angefangen, worbei aber sehr wenige Leuthe

\footnotetext{
1 SEIFERT, Herbert. Barock. In: Wien Musikgeschichte. Von der Prähistorie bis zur Gegenwart. Eds. Fritz-Hilscher, Elisabeth - Kretschmer, Helmut. Münster-Berlin: Lit 2011, S. 143-212, hier S. 182 f.

2 Ibid., S. 209 f. Ibid., 210.
} 
erschinen. ${ }^{" 4}$ Auch die Frequenz der Konzerte war anfangs noch unregelmäßig, doch bildeten die Wiener Fastenkonzerte bald eine willkommene zusätzliche Einnahmequelle für den permanent verschuldeten Impresario der Hoftheater. Auf dem Programm standen geistliche Vokalwerke, Instrumentalkonzerte und Sinfonien, aber auch Musik aus Opern wurde in Wien (im Gegensatz zu den Concerts spirituel in Paris) gebracht. ${ }^{5}$

Einen entscheidenden Impuls erhielt die Oratorien-Pflege in Wien mit der Gründung der Tonkünstler-Societät, mit deren Aufführungen sich das ursprünglich im geschlossenen höfischen Rahmen produzierte Oratorium nun öffentlich präsentierte. Doch die Verbindungen zum Hof und der höfischen Tradition der Gattung waren anfangs noch sehr eng: Komponisten und Ausführende kamen aus der Hofmusikkapelle bzw. deren Umkreis, die Aufführungen fanden unter Anwesenheit von Mitgliedern des Kaiserhauses und des Hofadels statt - nur der Aufführungsort war nicht mehr die exklusiven Kreisen vorbehaltene Hofburgkapelle, sondern das Theater nächst der Burg bzw. (v. a. in den Anfangsjahren) das Theatern nächst dem Kärntnerthor.

\section{Die Tonkünstler-Societät}

Seit dem Mittelalter war die Versorgung von Witwen und Waisen der Angehörigen einzelner Berufsgruppen durch religiös fundierten Zechen und Bruderschaften abgesichert gewesen - man denke an die bereits seit 1281 nachweisbare Nikolai-Bruderschaft (der städtischen Musiker) an St. Michael in Wien oder die ebendort angesiedelte 1725 gegründete Cäcilien-Bruderschaft der Hofmusiker ${ }^{6}$ -, die nicht nur „Berufsverbände“ darstellten, sondern vor allem im Sinne der alten Zechen und Zünfte der Handwerker als Solidargemeinschaften fungierten. Die zunehmende Säkularisierung des öffentlichen Bereiches ab der Mitte des 18. Jahrhunderts führte jedoch allmählich zu einer Zurückdrängung des religiösen Einflusses und der Schaffung eines durch landesherrliche Behörden kontrollierten Vereinswesens der erstarkenden Zivilgesellschaft. ${ }^{7}$

4 GROSSEGGER, Elisabeth. Theater, Feste und Feiern zur Zeit Maria Theresias 1742-1776. Nach den Tagebucheintragungen des Fürsten Johann Joseph Khevenhüller-Metsch, Obersthofmeister der Kaiserin. Wien: Verlag der ÖAW 1987 (Veröffentlichungen des Instituts für Publikumsforschung 12), S. $40 \mathrm{f}$.

5 EYBL, Martin - FRITZ-HILSCHER, Elisabeth. Vom Barock zur Wiener Klassik. In: Wien Musikgeschichte. Von der Prähistorie bis zur Gegenwart. Eds. Fritz-Hilscher, Elisabeth Kretschmer, Helmut. Münster-Berlin: Lit 2011, S. 213-269, hier S. 230 f. [Eybl], und detailreich bei: MORROW, Mary Sue. Concert Life in Haydn's Vienna: Aspects of a Developing Musical and Social Institution. Stuyversant: Pendragon Press 1989 (Sociology of music 7), S. 37-49.

6 Siehe SCHÜTZ, Karl. Musikpflege an St. Michael in Wien. Wien: Verlag der ÖAW 1980 (Veröffentlichungen der Kommission für Musikforschung 20), S. 50-54 [Nikolai-Bruderschaft] bzw. S. 76-81 [Cäcilienbruderschaft der Hofmusiker].

7 „Der absolutistische Staat des 18. Jahrhunderts beabsichtige, alle von ihm autonomen In- 
Die 1771 gegründete und per Dekret vom 23. 2. 1771 genehmigte Tonkünstler-Societät war eine der ersten Vereinigungen modernen Typus'. ${ }^{8}$ Dass sie vom Kaiserhaus nicht nur toleriert, sondern geradezu auffällig gefördert wurde, hatte einen praktischen Grund: Die Societät und ihr Pensionssystem entbanden den Hof bzw. die Dynastie von ihrer traditionellen Fürsorgepflicht gegenüber dienstunfähig gewordenen Hofbediensteten und deren Angehörigen. ${ }^{9}$ Dementsprechend drang der Hof auch darauf, dass die Mitglieder der Hofmusikkapelle auch Societäts-Mitglieder werden sollten. Maria Theresia stattete den Fonds mit $500 \mathrm{fl}$ aus, denen Joseph II. bald weitere $200 \mathrm{fl}$ hinzufügte. 32 Musiker der Hofkapelle waren auf Befehl der Kaiserin als Gründungsmitglieder aufgenommen worden; für diese zahlte die Herrscherin aus der Hofkassa jährlich $1800 \mathrm{fl}$ in die Kassa der Tonkünstler-Societät ein ${ }^{10}$ (d.h. deren Mitgliedsbeiträge übernahm der Hofärar, der sich so sehr kostensparend von allen Versorgungspflichten gegenüber diesen Musikern und deren Angehörigen ,freikaufte“). ${ }^{11}$

An der Spitze der Tonkünstler-Societät stand ein Protektor, der im Falle dieser Gesellschaft nicht durch ein Mitglied des Kaiserhauses gestellt wurde, sondern

stitutionen und Personenvereinigungen weitgehend zurückzudrängen bzw. zu unterbinden. Die weitgehende Unterdrückung aller vom Staate autonomen Personenvereinigungen wurde schließlich auch durch das Hofreskript vom 25. 8. 1764 bekräftigt. Dieses bestimmte, daß einzig der Staat über die Zulässigkeit von Vereinigungen durch Bewilligung oder Bestätigung für jeden Sonderfall zu entscheiden habe." PETE, Claudia. Geschichte der Wiener Tonkünstler-Societät. Wien: phil. Diss. 1996 [ungedruckt], S. 2.

8 Älter ist (im Bereich der habsburgischen Länder) nur die 1758 gegründete MedicinischerFacultät Wittwen-Sustentations-Kassa, die in ihrem Pensionssystem als direktes Vorbild für die Tonkünstler-Societät gelten kann. Vgl. dazu die Statuten in SCHÖPFER, Gerald. Sozialer Schutz im 16.-18. Jahrhundert. Graz: Leykam 1976 (Grazer Rechts- und Staatswissenschaftliche Studien 33), S. 232-235.

9 Vgl. dazu KUBISKA, Irene. „Zwischen Anspruch und Gnade“-Die Supplikationen Wiener Hofbediensteter an den Kaiser in der Mitte des 18. Jahrhunderts. Wien: Masterarbeit 2011, S. 31: „Gemäß dem Alimentationsprinzip, das den Herrscher zu einer Versorgung jener verpflichtete, die ihm lebenslang gedient hatten, konnten langjährige Bedienstete auf eine Altersversorgung hoffen." Zu den komplexen Möglichkeiten von Pension, Gnadengeldern, Spitalspfründen und der Versorgung von Witwen und Waisen durch den Hof bis zur Mitte des 18. Jahrhunderts vgl. ibid., S. 31-46.

PETE, op. cit., S. 29.

11 Ab dem Zeitpunkt, als die Tonkünstler-Societät ihre Tätigkeit aufnahm, wurde bei Ansuchen von Angehörigen von Mitgliedern der Hofmusikkapelle um Beteilung mit Gnadengelder genau durch das Obersthofmeisteramt geprüft, ob nicht ohnehin eine Versorgung durch die Societät gewährt wurde. Nur in Fällen, in denen keine Witwen- oder Waisenrenten aus der Societäts-Kassa ausgeschüttet wurde, wurden die Ansuchen durch den Obersthofmeister weitergeleitet und war die Zuteilung einer kaiserlichen Gnadengabe möglich (auch das Ansuchen von Konstanze Mozart nach dem Tod Wolfgangs wurde dahingehend geprüft, aber letztlich auch, da Mozart nicht Mitglied der Tonkünstler-Societät war, positiv entschieden). Vgl. Mozart. Dokumente seines Lebens. Gesammelt und erläutert von Deutsch, Otto Erich. Basel-London-New York: Bärenreiter 1961 (Wolfgang Amadeus Mozart, Neue Ausgabe sämtlicher Werke X/34), S. 378. 
aus den Reihen des Hofadels besetzt wurde. Auch die Amtsinhaber des Protektorates $^{12}$ zeigen die enge Verbindung zum Hof und in die Hofmusikkapelle hinein und akzentuieren diese; die enge Verbindung zwischen Obersthofmeisteramt und Societät - ab 1791 in Personalunion von Protektor und Cavagliere della musica - hatte durchaus praktische Gründe, da sich sowohl bei der Behandlung von Gnadengesuchen an den Hof als auch, wenn es um die Bereitstellung und Adaption der Hoftheater für die Akademien ging wie um deren Abrechnungen, die Amtswege so vereinfachten und verkürzten. Auch Hofkapellmeister und Vizekapellmeister wurden in den Ämtern von Präses und Vize-Präses der Societät gleichsam „gespiegelt“; v. a. Gassmann, Bonno und Salieri nahmen diese Aufgaben sehr ernst und engagierten sich in den Akademien durch zahlreiche Beiträge von Originalkompositionen wie als Dirigenten der Konzerte.

Der Tonkünstler-Societät gehörten 54 Gründungsmitglieder an. „Bereits nach zehn Jahren erreichte der Mitgliederstand eine Zahl von 104. In den ersten hundert Jahren seit Bestehen des Vereines waren demselben 405 Mitglieder beigetreten. Bis zur Vereinsauflösung 1939 waren 662 Musiker in die Societät aufgenommen worden. "'13 Aufnahmebedingungen waren - abgesehen von einem tadellosen Ruf ${ }^{14}$ - die Vorlage eines Taufscheins ${ }^{15}$ ein Wohnort in Wien ${ }^{16}$ und der Erlag eines einmaligen Eintrittsgeldes sowie eines ,quartaliter“ (d.h. vierteljährlich) zu entrichtenden Mitgliedsbeitrages. Mitglieder der kaiserlichen Hofmusikkapelle oder andere prominente Musiker der Zeit wurden meist problemlos aufgenommen, Mitglieder weniger bekannter Orchester oder den Mitgliedern des Vereines (und

12 „Der Protektor war einerseits Schutzherr und Förderer, dessen Verbindungen manch langwierigen Instanzenweg abkürzen und Entscheidungen bezüglich des Vereins positiv beeinflussen konnten. Andererseits sollte er Gewähr dafür bieten, daß der Verein die erlaubten Grenzen nicht überschritt." OBROVSKI, Herta. Das Wiener Vereinswesen im Vormärz. Wien: phil. Diss. 1970, zitiert bei PETE, op. cit., S. 25. Dem ersten Protektor, Johann Wenzel Graf Sporck folgten Johann Joseph Fürst Khevenhüller-Metsch, Joseph Adam Johann Nepomuk Fürst Schwarzenberg, Georg Adam Fürst Starhemberg, Wolf Franz Xaver Fürst Rosenberg; dessen Nachfolger wurde 1791 Johann Wenzel Graf Ugarte, der auch die Position des Cavagliere della Musica am Hof innehatte. In der Folge wurden diese beiden Ämter fast automatisch verbunden. Ugarte folgten die Hofmusikgrafen Johann Ferdinand Graf Kuefstein, Moriz Johann Joseph Graf Dietrichstein, Carl Leonhard Graf Harrach, Thaddäus Graf Amadé und Leopold Graf Podstatzky-Liechtenstein nach. 1849 bis 1863 blieb diese Stelle unbesetzt; mit der Umwandlung in den „Haydn-Verein“ wurde der Protektor erstmals frei gewählt (Franz Graf Kuefstein) - vgl. PETE, op. cit. S. 26.

13 PETE, op. cit., S. 4.

14 Tanzmusiker beispielsweise wurden nicht aufgenommen (aus diesem Grund wurden die Ansuchen von Joseph Lanner und Philipp Fahrbach abschlägig beschieden), ebenso hatten herumreisende Virtuosen keine Chance. Vgl. dazu ibid., S. 33-34 bzw. S. 55-56 (Statuten).

15 An dieser „Hürde“ scheiterte beispielsweise die Aufnahme von Wolfgang Amadeus Mozart, dem es (wohl aus Gründen persönlicher Desorganisation) nicht gelang, vom Datum des Ansuchens um Aufnahme in die Societät (11. Februar 1785) bis zu seinem Tod einen solchen aus Salzburg zu bekommen und vorzuweisen (dazu ibid., S. 51-54).

16 Nur zwischen 1807 und 1862 war es auch Musikern von Außerhalb möglich, in die Tonkünstler-Societät aufgenommen zu werden (vgl. ibid., S. 55 f.). 
v.a. dem Vorstand) unbekannte Musiker mussten sich einer Aufnahmsprüfung unterziehen. Doch weniger an der Aufnahmsprüfung als an der Höhe des Einmalerlages und der regelmäßig zu entrichtenden Mitgliedsbeiträgen scheiterten viele Mitglieder, denn diese waren, um die Auszahlung der Pensionen sicherstellen zu können, in für Mitglieder weniger prominenter Orchester und Theater als jener des Hofes nahezu unerschwinglicher Höhe. ${ }^{17}$

1862 wurde die Tonkünstler-Societät in Haydn-Verein umbenannt und widmete sich fortan der Pflege der beiden späten Oratorien Josephs Haydn, Die Schöpfung bzw. Die Jahreszeiten, die jährlich zur Aufführung gebracht wurden; die ursprüngliche Funktion als Pensionsvereines blieb jedoch auch weiterhin erhalten. $1939^{18}$ wurde der Haydn-Verein im Zuge der Gleichschaltung nach dem Anschluss Österreichs an das Deutsche Reich aufgelöst.

\section{Die Akademien}

Die ab 1772 zweimal jährlich veranstalteten Akademien der Tonkünstler-Societät zählen zu den ältesten kontinuierlichen öffentlichen Konzertreihen Wiens. ${ }^{19}$ Die Societät war hier „,das erste und blieb auch lange Zeit das einzige Musikinstitut, welches sich in erster Linie der Pflege der großen Oratorienwerke widmete". Dass die Oratorien nicht mehr im semi-liturgischen Raum und Ort und in der Geschlossenheit des Hofes aufgeführt wurden, wie dies seit der Einführung dieser Gattung üblich war, sondern im Rahmen einer Konzert-Akademie in einem der Hoftheater, war ebenfalls neu, ebenso die Wandlung von der quasi-religiösen Handlung zum Konzert, bei dem doch wohl mehr „diletto“ als innig-religiöse Betrachtung im Vordergrund stand. Als einzige Anbindung an den ursprünglichen liturgischen Konnex blieb der Zeitpunkt der Akademien: in der Karwoche bzw. wenige Tage vor Weihnachten, als Anbindung an den Hof die Ausführung durch Mitglieder der Hofmusikkapelle bzw. der Hoftheater, meist unter Leitung des Hofkapellmeisters (und meist auch die Anwesenheit des Kaisers bzw. von Mitgliedern des Kaiserhauses bei den Akademien). ${ }^{20}$

17 Selbst die Musiker des Kärntnerthor-Theaters konnten sich - abgesehen von den Spitzenkräften - diese Beträge nicht leisten, weshalb sie ein eigenes System der Versorgung schaffen mussten. Die in Nachahmung der großen Akademien der Tonkünstler-Societät 1842 geplanten Philharmonischen Abonnement-Konzerte wurden - aus der Not geboren - zum heute höchst erfolgreichen Unternehmen Wiener Philharmoniker (zur Gründung der Wiener Philharmoniker vgl. HELLSBERG, Clemens. Demokratie der Könige. Die Geschichte der Wiener Philharmoniker. Zürich-Wien-Mainz: Kemayr \& Scheriau 1992, S. 14.

Der Haydn-Verein wurde auf Anordnung des Stillhaltekommissars vom 9. März 1939 als Rechtsperson aufgehoben und dem Deutschen Ring österreichischer Lebensversicherungs $A G$ eingegliedert. Mit 18. April 1939 wurde der Verein aus dem Vereinskataster gelöscht (PETE, op. cit., S. 31).

20 Die Wahl dieser Konzerttermine sollte jedoch weniger unter liturgischen als praktischen Gründen gesehen werden. Es handelt sich bei beiden Daten um sogenannte Norma-Tage, an 
Der Beschluss, um die Bewilligung von jährlich zwei großen Akademien an spielfreien Tagen in der Fastenzeit und im Advent im Kärntnerthor-Theater anzusuchen, wurde am 17. April 1771 einstimmig gefasst und mit 20. November desselben Jahres durch den Hof bewilligt. Hauptaspekt dieser Akademien war eine Erhöhung des Vereinskapitals, da diese Akademien reine Benefiz-Konzerte waren, bei denen die Mitglieder der Societät verpflichtet waren, nicht nur anwesend zu sein, sondern auch unentgeltlich zu musizieren. ${ }^{21}$ Die Nähe zum Hof und der Hofmusikkapelle wie die Möglichkeit, in einem öffentlichen Konzert mit den renommiertesten Musikern der Stadt auftreten zu können, ließ die Akademien auch für neuangekommene Musiker, die sich erst in der Stadt etablieren mussten, höchst attraktiv erscheinen. So schreibt Wolfgang Amadeus Mozart am 24. März 1781 an seinen Vater in Salzburg: „- O, hätte ich gewust, daß ich die fasten nach Wienn kömmen würde, hätte ich ein kleines oratorio geschrieben, und zu meinen vortheile im theater gegeben, wie es hier alles macht -ich hätte leicht vorher zu schreiben gehabt, weil ich die stimmen alle kenne; - wie gerne gäb ich nicht ein öfentliches Concert wie es hier der Brauch ist, aber - es wird mir nicht erlaubt, das weis ich gewis, denn, stellen sie sich vor - sie wissen daß hier eine Societet ist, welche zum vortheile der Witwen von den Musicis accademien giebt - alles was nur Musik heist spiellt da umsonst - das orchestre ist 180 Personnen stark - kein virtuos der nur ein bischen liebe des Nächsten hat, schlägt es ab darin zu spiellen, wenn er von der Societet aus darum ersuchet wird - denn, man macht sich auch sowohl beym kayser als beym Publicum darum beliebt. - starzer hatte den auftrag mich darum zu bitten, und ich sagte es ihm gleich zu, dich müste ich vorher meines fürsten Gutachten darüber vernehmen - und ich hatte gar keinen zweifel weil es eine geistliche art, und unentgeldlich nur um eines gutes Werk zu thun, ist; - er erlaubte es mir nicht; - die ganze noblesse hier hat ihm dieses übel genommen. - mir ist es nur wegen diesem leid; - ich hätte kein Concert, sondern /:weil der kayser in der Proscen loge ist:/ ganz allein /:die gräfin thun hätte mir ihr schönes steiner=Pianforte darzu gegeben:/ Preludirt, eine fuge - und dann die variationen je suis lindor gespiellt. - wo ich noch das so öfentlich gemacht habe, habe ich den grösten beyfall erhalten - weil es so gut gegen einander absticht, und weil Jeder - was hat; aber Pazienza; - "22 Die

denen in den Theatern keine Aufführungen stattfinden durften und somit nicht nur der Raum, sondern auch das Personal zur Verfügung standen.

21 Für die Mitglieder der Gründergeneration scheint die aktive Teilnahme bei den Akademien eine Selbstverständlichkeit gewesen zu sein, denn erst 1807 wird in den Statuten verankert, „daß jedes Mitglied bei den Proben und Akademien erscheinen müsse. Die abwesenden Mitglieder sollten anstatt ihres Dienstes einen jährlichen Akademiebeitrag von 12 Gulden zahlen. Wenn ein Mitglied bei einer Probe oder Produktion ohne Ursache wegblieb, so sollte es das erste Mal erinnert, das zweite Mal mit Ausschliessung bedroht und das dritte Mal ausgeschlossen werden. Laut den Statuten von 1862 war ein Mitglied verpflichtet, bis zum sechzigsten Lebensjahr bei den Akademien mitzuwirken." PETE, op. cit., S. 66. gesammelt und erläutert von Deutsch, Otto Erich. Band III: 1780-1786. Kassel-Basel-London: Bärenreiter 1987, Nr. 585, S. 97-101, hier S. 99. 
„Pazienza“ machte sich bald bezahlt, denn am 3. April 1781 trat Wolfgang Amadeus Mozart schließlich doch in einer Akademie der Tonkünstler-Societät auf und konnte sich mit einer Symphonie und einem von ihm selbst gespielten Solo auf dem Fortepiano präsentieren. Am 22. Dezember 1783 durfte Mozart mit einem seiner Klavierkonzerte erneut auftreten, und am 13. März 1785 wurde sein $D a$ vidde penitente im Rahmen einer Societäts-Akademie uraufgeführt (vgl. dazu die Daten im Anhang) - kurz zur (datiert mit 11. Februar 1785) hatte Mozart sein Aufnahmegesuch bei der Societät eingereicht.

Nicht nur Mozart wollte die Akademien der Tonkünstler-Societät als Präsentations-Forum nützen; wie aus der Auflistung im Anhang hervorgeht, wurden die Oratorien, die den Kern der Akademien der Tonkünstler-Societät bildeten, von Konzerten einheimischer Virtuosen oder neu hier angekommener, die sich in Wien präsentieren und etablieren wollten, umrahmt. Und möglichst vielen Künstlern Gelegenheit zum Auftritt bieten zu können, wechselten diese „umrahmenden" Programm-Teile bei den nach wenigen Tagen üblichen Wiederholungen der Oratorienaufführungen.

Die Akademien, die ab circa 1800 immer stärker den Aspekt der Pflege der beiden Haydn-Oratorien Die Schöpfung und Die Jahreszeiten in den Mittelpunkt rückten, wurden erst 1872 - nach genau 100 Jahren - ausgesetzt. „Mit Erlass vom 26. April 1873 wurde eine Abänderung des $\$ 43$ [der Statuten] genehmigt. Bezüglich der Veranstaltung von Akademien wurde nun hinzugefügt, daß diese unterbleiben könnten entweder auf höchsten Befehl oder nach Ermessen des Ausschusses und mit hoher Genehmigung gegen angemessene Entschädigung oder wenn die Veranstaltung nicht als vorteilhaft für den Verein erschiene. Für die Zeit, innerhalb welcher die Mitwirkung der Vereinsmitglieder nicht in Anspruch genommen wurde, entfiel auch die Entrichtung des Akademiebeitrages. ${ }^{\text {"23 }}$

\section{Zum Programm der Akademien der Tonkünstler-Societät}

Bereits ein halbes Jahr nach dem Beschluss zur Abhaltung von Akademien fand die erste im Kärntnerthor-Theater statt. Das Theater nächst dem Kärntnerthor blieb bis März 1783 Veranstaltungsort der Akademien, ab dem Weihnachtskonzert 1783 fanden sie (von wenigen Ausnahmen abgesehen) im Theater nächst der Burg statt. ${ }^{24}$

Bereits ab 1772 ist ein hoher Grad an Konsequenz sowohl in der Frequenz der Konzerte wie in deren inhaltlicher Planung zu bemerken. Die Konzerte fanden entweder in der Karwoche statt oder in der Woche vor Weihnachten; jedes Konzert wurde mindestens einmal wiederholt (meist 2 bis 3 Tage später) - nur das Adventkonzert 1780 und das Karwochenkonzert 1790 mussten aufgrund der

23 PETE, S. $77 \mathrm{f}$.

24 Vgl. dazu die Tabelle im Anhang. 
Landestrauer nach dem Tod Maria Theresias bzw. Josephs II. abgesagt werden ${ }^{25}$ Im Zentrum der Aufführung stand ein großes Oratorium - anfangs in italienischer Sprache (nach Texten von Pietro Metastasio oder von Librettisten in der Metastasio-Nachfolge), ab 1779 auch zunehmend mit deutschen Texten, doch dominieren bis in die 1790er Jahre die italienischen Texte. Auch die deutschen Texte waren meist Übersetzungen bzw. Nachdichtungen traditioneller italienischer Oratorien-Sujets (beispielsweise 1779 Die Israeliten in der Wüste oder 1781 Die Pilgrime auf Golgotha wie 1794 Die Hirten bei der Krippe zu Bethlehem) und orientieren sich in der Art der Aufbereitung und Darstellung des Themas am barocken italienischen Oratorium; sie haben keine Handlung in engeren Sinn, sondern beschreiben in den Arientexten v. a. Seelenzustände und Empfindungen der Akteure in Zusammenhang mit einem meist biblischen Ereignis.

Wie die folgende grobe Einteilung der aufgeführten Oratorien bzw. Kantaten wie die Gesamtliste im Anhang zeigen, dominierten vor allem alttestamentarische Themen sowie Texte mit Bekennerthematik; insgesamt zeigt die Auflistung noch eine starke Verwurzelung in der barocken italienischen Librettistik der Arcadia. Erst mit den beiden Haydn-Oratorien Die Schöpfung und Die Jahreszeiten wird diese Dominanz um 1800 nachhaltig gebrochen. Joseph Eyblers Die Hirten bei der Krippe zu Bethlehem gehen zwar musikalisch-stilistisch neue Wege und nehmen Vieles, was als Innovation Haydns in der Schöpfung gilt, voraus, ${ }^{26}$ bleiben aber von der dramaturgischen Anlage der traditionellen italienischen Form noch treu; die traditionellen Wege auch in der dramaturgischen Anlage werden erst im zweiten großen Werk Eyblers für die Tonkünstler-Societät, Die vier letzten Dinge (1810), endgültig verlassen: Hier präsentiert sich eine eigene Gattungsform des deutschsprachigen (Wiener) Oratoriums, mit der sich Eyblers Werk in eine Reihe mit denen von Joseph Haydn, Peter von Winter oder Abbé Vogler stellt.

251791 musste wegen einer offenbar sehr kurzfristigen und nicht durch einen „Einspringer“ kompensierbare Ausfall eines erkrankten Sängers das Adventkonzert und dessen Wiederholung abgesagt werden; auch im Jahr darauf erkrankte eine am Adventkonzert führend mitwirkende Sängerin, doch konnte 1792 wenigstens die erste Aufführung noch stattfinden (vgl. die Tabelle im Anhang).

26 Die beginnt schon in der Ouvertüre mit einer an das „Chaos“ in der Schöpfung erinnernden Darstellung von Dunkelheit und Sonnenaufgang (Eyblers Werk entstand 1794, Haydns Schöpfung jedoch erst 1796 bis 1798), setzt sich in den Arien fort, die sich stilistisch von der opera seria emanzipiert haben und einen eigenen, neuen, „oratorischen“ Stil suchen, und ist vor allem in den Chören zu finden, die nicht nur wesentlich mehr Platz beanspruchen als im italienischen Oratorium, sondern deutlich auf eine intensive Händel-Rezeption hinweisen. Vgl. dazu die Handschriften in der Musiksammlung der Österreichischen Nationalbibliothek in Wien (A-Wn, Mus.Hs. 3231/1-2: Die Hirten bei der Krippe zu Bethlehem. [Wien] 1794, Partitur, Handschrift, 2 Bände, 240 fol.; - A-Wn, Mus.Hs. 19.212: Die vier letzten Dinge. Ein Oratorium in drey Abtheilungen. Wien 1810, Partitur, Handschrift, XII + 178 fol.). 


\section{Tab. 1: Oratorienstoffe (1772-1810):}

1) alttestamentarisch:

La betulia liberata

1772, 1776

Ester

1773

Giuseppe riconosciuto

1774

Il cantico di tre fanciulli

1774

Il ritorno di Tobia

Davidde (il) penidente

1775, 1777 (in Teilen), 1784, 1808

1775,1785

Isacco figura del Redentore

1776,1782

Judas Maccabäus

1779,1806

Die Israeliten in der Wüste

1779,1783

Giobbe

Gioas (Re di Giuda)

1786, 1789 (in Teilen)

1786,1795

Mose in Egitto

1787,1790

Die Schöpfung

1799, $1800(2 x), 1804,1805,1807,1809,1810$

\section{2) neutestamentarisch:}

a) Passion und Auferstehung

Santa Elena al Calvario

La Passione di Giesu Cristo

$1772,1773,1781$

La Passione del Redentore

1777

1778

Die Pilgrime auf Golgotha

1781

I Profeti al Calvario

1787

La morte, e la deposizione dalla croce di Gesu Cristo 1788

Die sieben Worte unseres Erlösers [...]

Das heilige Grab

$1798,1799,1801$

1803

b) andere Stoffe

Der verlorene Sohn

1780

Die Hirten bei der Krippe zu Bethlehem

1794

Die vier letzten Dinge

1810

\section{3) sonstige:}

Alcide al bivio

1781 (in Teilen?)

Ifigenia in Tauride

Venere ed Adone, Kantate

1784 (in Teilen?)

Timotheus, oder die Gewalt der Musik

1792,1793

1796,1797

La Riconoscenza, Kantate

1796

Der Retter in Gefahr, Kantate

1796, 1797 (in Teilen)

Die Jahreszeiten

$1801,1802(2 \mathrm{x})$,

$1804,1808,1809$

Kastor und Pollux (Castore e Polluce), Kantate

1803

Endimione e Diana, Kantate

1807 
Umrahmt bzw. eingeleitet werden die Oratorien durch Symphonien und/oder Instrumentalkonzerte. Nur selten sich die bis weit in das 19. Jahrhundert in öffentlichen Konzerten und Akademien so beliebten „Mischprogramme“ mit Arien, Chören, Symphonien bzw. Symphoniesätzen und Instrumentalkonzerten (gehäuft allerdings in den 1780er Jahren) zu finden, wobei auch bei diesen Programmen oft neue „Cantaten“ aufgeführt wurden (auch Mozarts Davidde penitente ist im Zuge eines solchen Konzertes aufgeführt worden).

In den Namen der Komponisten der Oratorien (die von diesen oft auch der Tonkünstler-Societät gewidmet wurden) spiegelt sich der Generations- wie Stilwechsel zwischen Spätbarock und Klassik (bis hin zu Biedermeier und Frühromantik) wider. Die meisten von ihnen waren hauptsächlich in Wien tätig, viele Mitglieder der kaiserlichen Hofmusikkapelle bzw. befanden sich in einem Naheverhältnis zum Hof bzw. den Hoftheatern:

\section{Tab. 2: Komponisten der Oratorien der Akademien und Häufigkeit (1772-} 1810)

(Reihung chronologisch nach der Aufführung ihrer Werke in den Akademien)

Florian Leopold Gassmann: 2

Johann Adolph Hasse: 4

Carl Ditter von Dittersdorf: 3

Giuseppe Bonno: 1

Joseph Haydn: 21

Ferdinand Bertoni: 1

Antonio Salieri: 1

Joseph Starzer: 1

Georg Friedrich Händel: 2

Maximilian Ulbrich: 2

Johann Georg Albrechtsberger: 1

Marianne Martinez: 1

Anton Teyber: 1

Leopold Koželuh: 2

Domenico Mombelli: 1

Joseph Eybler: 2

Antonio Casimir Cartellieri: 1

Peter Winter: 2

Ferdiando Paer: 1

Abbé Vogler: 1

Verfolgt man über 30 Jahre die Konzerte, so tritt der zweifache Generationswechsel klar zutage: von der noch aus der spätbarocken Tradition schöpfenden Komponisten wie Hasse oder Bonno, über die Komponistengeneration des Übergangsstils vom Barock zur Klassik - sie dominiert die Programm bis zum Ende der 1780 Jahre -, bis hin zur jungen Generation, die bereits für die Weiterent- 
wicklung der Klassik in Richtung Romantik und Biedermeier steht (wie Eybler, Preindl, Joseph Weigl, Koželuh bzw. als Instrumentalkomponist Beethoven). Stehen die Werke der Komponisten der ersten Generation (wie Gassmann, Hasse, Bonno, Dittersdorf, aber auch Haydn mit Il ritorno di Tobia) ganz unter dem Einfluss der spätbarocken opera seria, lassen sich an den Werken der folgenden Generationen die aktuellen Entwicklungen von der Dominanz des Opernstils hin zu Symphonie und Konzert, aber auch zu Accompagnato wie der schlichten, liedhaften Gestaltung der Arien der Singspiele bis hin zu Elementen des Frühbiedermeier beobachten; dennoch bleibt (rein formal) die da capo-Form für die Arien bis zum Ende des 18. Jahrhunderts vorherrschend. Der Wandel vom italienischen Oratorium zu einem eher nach englischer Tradition ausgerichteten eigenständigen deutschsprachigen wurde erst ab den späten 1780er Jahren konsequent verfolgt, obwohl Händels Oratorien in Wien schon zu Lebzeiten des Komponisten bekannt gemacht wurden und sich regelmäßig in den Konzertberichten finden; es sind auch vorerst die grobformalen dramaturgischen Raster, die Aufwertung des Chores und deren musikalische Gestaltung, die in die Oratorien-Produktion für die Tonkünstler-Societät übernommen wurden, in den Stoffen und der dramaturgischen Gestaltung der Libretti, der Wahl der Topoi wie Verwendung eines reglementierten Vokabulars wirkte jedoch noch lange die Tradition der Arcadia und ihres Wiener Hauptexponenten Pietro Metastasio nach.

Abschließend sei eine Beantwortung der im Titel gestellten Frage versucht: Nach dieser sehr stark verdichteten Darstellung der Fragestellung kann weder eindeutig in die eine, noch in die andere Richtung argumentiert werden, vielmehr finden sich verbindende wie überlappende Aspekte, deren Anteile sich allmählich hin von einem Überwiegen traditioneller Elemente hin zu einem in Gattung, Stil wie Aufführungskontext neuen Produkt verschieben. Die Oratorien-Produktion der Tonkünstler-Societät ist in der Verwendung der Stoffe und bis in die 1780er Jahre hinein wie auch in der formalen und dramaturgischen Gestaltung in der direkten Nachfolge der höfischen Oratorienpflege des spätbarocken Hofes zu sehen; erst allmählich erfolgte, parallel mit der Entwicklung der Gattungen in der zweiten Hälfte des 18. Jahrhunderts in Wien, auch eine Neuformulierung des Oratoriums - sowohl in der Wahl der Stoffe, deren dramaturgischer Aufbereitung sowie der musikalisch-kompositorischen Umsetzung der Texte. Als Kontrapunkt zum höfischen Oratorium ist in erster Linie die neue Funktion, die die OratorienAufführungen der Tonkünstler-Societät von Anfang an hatten, zu sehen: Sie sind öffentliche Konzerte und auf pekuniären Erfolg ausgerichtet; die Demonstrierung habsburgischer pietas und humilitas im geschlossenen Rahmen des Hofes und der Hofkapelle wurde gegen ein Konzert, das in erster Linie Vergnügen und Zerstreuung diente (wenngleich der geistliche Aspekt durch die Wahl der Termine nicht ganz außer Acht gelassen wurde) getauscht. Der gesellschaftlichen Zutrittsschranke war einer wirtschaftlich-monetäre, die Finanzierbarkeit eines Eintrittsbilletts, gewichen. Als Verbindungsglied zwischen Tradition und Kontrapunkt fungierten die Mitglieder der Societät selbst, die vorwiegend Musiker der Hof- 
musikkapelle bzw. der Hoftheater waren, und an deren Spitze der Hofkapellmeister, der oft auch als Dirigent der Akademien fungierte, stand. Erst ab 1800, mit der Dominanz der beiden großen Haydn-Oratorien in den Konzertprogrammen der Tonkünstler-Societät ändert sich deren Funktion: sie steht nun nicht mehr im Fokus zeitgenössischer Oratorienproduktionen, sondern widmet sich nun immer stärker der Pflege einer Tradition und der Entwicklung des Wertekanons des 19. Jahrhunderts - ihren bahnbrechend innovativen Geist, sowohl in künstlerischer Hinsicht, wie auch in sozialer und rechtlicher, verlor die Tonkünstler-Societät ab 1810 jedoch sukzessiv.

\section{ANHANG}

Die Konzerte der Tonkünstler-Societät 1772 bis $\mathbf{1 8 0 0}^{27}$

(Konzertort - wenn nicht anders angegeben - war das Theater nächst dem Kärntnerthor)

29. 3. 1772; Wiederholungen: 1. 4./ 5. 4. 1772

Joseph Starzer: Symphonie

Florian Leopold Gassmann: La betulia liberata

Franz Aspelmayr: Symphonie (29. 3. / 5. 4.)

Konzert für Flöte (1. 4.)

17. 12. 1772; Wiederholung: 20. 12. 1772

Johann Adolph Hasse: Santa Elena al Calvario

Konzert für Violoncello (17. 12)

Konzert für Klarinette (17. 2.)

Konzert für Viola (20. 12.)

21. 3. 1773; Wiederholung: 25. 3. 1773

Johann Adolph Hasse: Santa Elena al Calvario [mit den selben Solisten wie 1772]

Konzert für Klarinette (21. 3.)

Konzert für Oboe (25. 3.)

19. 12. 1773; Wiederholung: 21. 12. 1773

Carl Ditter von Dittersdorf: Ester

Konzert für Violine (19. 12.)

Konzert für Flöte (21. 12.)

20.3.1774 [keine Wiederholung bekannt]

Giuseppe Bonno: Il Giuseppe riconosciuto

Konzert für Viola

18. 12. 1774; Wiederholung: 21. 12. 1774

Johann Adolph Hasse: Il cantico dei tre fanciulli

Konzert für Violine und Violoncello (18. 12.)

Konzert für Violoncello (21. 12.)

27 Die Daten wurden den Arbeiten von MORROW, op. cit., S. 237-364, entnommen. 
2. 4. 1775; Wiederholung: 4. 4. 1775

Joseph Haydn: Il ritorno di Tobia [unter der Leitung des Komponisten]

Konzert für Violine (2. 4.)

Konzert für Violoncello (4. 4.)

17. 12. 1775; Wiederholung: 19. 12. 1775

Ferdinand Bertoni: Davidde il penitente

Giovanni Battista Martini: Symphonia aus Henri IV. (17. 12.)

Konzert für Klarinette (19. 12.)

17. 3. 1776; Wiederholung: 20. 3. 1776

Carl Ditter von Dittersdorf: Isacco figura del Redentore

Konzert für Flöte (17. 3.)

Giovanni Battista Martini: Symphonia aus Henri IV. (20. 3.)

18. 12.1776 [keine Wiederholung verzeichnet]

Florian Leopold Gassmann: La Betulia liberata

Konzert für Oboe

17. 3. 1777

Carlo Ordonez: Symphonie

Joseph Haydn: Chor aus Il ritorno di Tobia

Traetta: Rezitativ und Cavatina aus Armida

Paisible: Violin-Konzert

Carl von Kohaut: Symphonie

Aria

Carl von Kohaut: Konzert für verschiedene Instrumente (Paisible)

Georg Christoph Wagenseil: Cantata [ohne genaue Titelnennung]

18. 12. 1777; Wiederholung: 21. 12. 1777

Antonio Salieri: La Passione di Giesu Cristo

Carlo Oronez: Symphonie (18. 12.)

Konzert für Violine (21. 12.)

23. 3. 1778; Wiederholung: 27. 3. 1778

Joseph Starzer: La Passione del Redentore

Konzert für Violine (23. 3.)

Konzert für Violine (27. 3.)

20. 12.1778

Johann Sperger: Symphonie

Carl Ditter von Dittersdorf: Aria [ohne nähere Bezeichnung]

Georg Friedrich Händel: großer Chor [ohne nähere Bezeichnung]

Sperger: Konzert für Kontrabass

Franz Teyber: Aria

Sacchini: großer Chor

Gottfried van Swieten [?]: Symphonie

Giordani: Aria

Händel: großer Chor

Konzert für Violine

Giuseppe Sarti: Trio für drei Stimmen

Händel: großer Chor 
21. 3. 1779; Wiederholung: 23. 3. 1779

Georg Friedrich Händel: Judas Maccabäus

Konzert für Oboe (21. 3.)

Josef Starzer: Konzert für 2 Orchester mit Trompeten und Trommeln (23. 3.)

19. 12. 1779; Wiederholung: 21. 12. 1779

Maximilian Ulbrich: Die Israeliten in der Wüste

Konzert für Violine (19. 12.)

Charles Janson: Konzert für Violoncello (21. 12.)

12. 3.1780

Bach [welcher?, Carl Philipp Emanuel?, Johann Christian?]: neue Symphonie

Monza: Aria

Georg Friedrich Händel: großer Chor

Joseph Starzer: Konzert für fünf Blasinstrumente

Tomasso Giordani: Aria mit Flöte und Gesang

Händel: großer Chor

Konzert für Violine

Friedrich Hartmann Graf [?]: Der verlorene Sohn [Kantate?]

14. 3. 1780 [mit neuem Programm!]

Joseph Haydn: neue Symphonie

Insanguine: Aria

Georg Friedrich Händel: großer Chor

Konzert für Violine

Ignaz Holzbauer: Aria

Antonio Sachini: Chor

Friedrich Hartmann Graf [?]: Der verlorene Sohn

Das Adventkonzert 1780 entfiel wegen der Landestrauer nach dem Tod von Maria Theresia.

11. 3. 1781; Wiederholung: 13. 3. 1781

Thaddäus Huber: Symphonie

Konzert für Violoncello (nur 11. 3.)

Konzert für Oboe (nur 13. 3.)

Johann Adolph Hasse: Alcide am Scheideweg (Alcide al bivio)

1. 4. 1781; Wiederholung: 3. 4. 1781

Georg Albrechtsberger: Die Pilgrime auf Golgotha

Wolfgang Amadeus Mozart: Symphonie (nur 3. 4.)

Forte-piano-solo (gespielt durch Mozart, nur 3. 4.)

22. 12. 1781; Wiederholung: 23. 12. 1781

Johann Adolph Hasse: Santa Elena al Calvario

17. 3. 1782; Wiederholung:19.3. 1782

Marianne Martinez: Isacco figura del Redentore

Duo für Oboe und Fagott (W. Kauzer, Triebensee; 17. 3.)

Konzert für Violine (J. Hofmann, 19. 3.)

22. 12. 1782; Wiederholung: 23. 12. 1782 [identes Programm], Theater nächst der Burg

Christoph Willibald Gluck: Symphonie 
Gluck: zwei Chöre

Naumann: Aria

Georg Friedrich Händel: Chor

Naumann: Aria

Cambini: Konzert für Violine

Georg Christoph Wagenseil: Kantate [ohne genaue Titelnennung]

Saccchini: Chor

Antonio Salieri: Aria

Händel: Chor

6. 4. 1783; Wiederholung: 8. 4. 1783

Maximilian Ulbrich: Die Israeliten in der Wueste

Harmoniemusik (durch die Musiker der Harmoniemusik des Hofes, 6. 4.)

Graf: Konzert für Flöte (8. 4.)

22. 12. 1783; Wiederholung: 23. 12. 1783, Theater nächst der Burg

Joseph Haydn: Symphonie

Haydn: Chor

Sacchini: Aria

Wolfgang Amadeus Mozart: Klavier-Konzert (gespielt durch Mozart, 22. 12.)

Konzert für Violine (23. 12.)

Koželuh: Symphonie

Mozart: Rondo für Singstimme (KV 431?)

Sarti: Trio für Gesang und Orchester

Johann Adolph Hasse: Chor

Sacchini: Chor

Dittersdorf: Chor

Dirigent und Leitung: Joseph Starzer

28. 3. 1784; Wiederholung: 30. 3. 1784, Theater nächst der Burg

Joseph Haydn: Il ritorno di Tobia [dirigiert durch Haydn]

Fischer: Konzert für Violine (28. 3.)

Kreyser: Konzert für Flöte (30. 3.)

22. 12. 1784; Wiederholung: 23. 12. 1784

Thaddäus Huber: Symphonie

Thomaso Traetta: Ifigenia in Tauride

Joseph Haydn: großer Chor

Antonio Salieri: Konzert für Oboe und Flöte (22. 12.)

Fodor: Konzert für Violine (23. 12.)

Georg Friedrich Händel: Chor (23. 12.)

13. 3. 1785; Wiederholung: 15. 3. 1785, Theater nächst der Burg

Joseph Haydn: neue Symphonie

Zwei Arien [ohne Nennung der/des Komponisten]

Florian Leopold Gassmann: Chor

Sacchini: Chor (15. 3.)

Haydn: Chor

Konzert für Oboe (13. 3.)

Konzert für Violine (15. 3.)

Wolfgang Amadeus Mozart: Davidde penitente 
8. 4. 1786; Wiederholung: 9. 4. 1786, Theater nächst der Burg

Joseph Haydn: Symphonie (8. 4.)

Thaddäus Huber: Symphonie (9. 4.)

Fränz(e)l: Violinkonzert (gespielt von Fränzel) (8. 4. nach Giobbe, 9. 4. vor Giobbe)

Carl Ditters von Dittersdorf: Giobbe (Teil 1: 8. 4., Teil 2: 9. 4.)

Ditters: Chor aus Giobbe (Teil 1)

22. 12. 1786, Wiederholung: 23. 12. 1786

Anton Teyber: Gioas (unter der Leitung von Teyber)

Cäsar Scheidl:Konzert für Pianoforte (gespielt von Scheidl; 22. 12.)

Violinkonzert (Joseph Zistler, 23. 12.)

30. 3. 1787, Wiederholung: 1. 4. 1787, Theater nächst der Burg

Dittersdorf: neue Symphonie

Wagenseil: neue Kantate mit Chor

Violinkonzert (Zeno Menzel, Philipp Schindlöcker, 30. 3.)

Flötenkonzert (Nikolaus Scholl)

V. Martín y Soler: Stücke aus Una cosa rara, arrangiert für die Harmoniemusik des Kaisers Joseph Gazzaniga: I Profeti al Calvario (Leitung: Umlauf, nur 30. 3.)

22. 12. 1787, Wiederholung: 23. 12. 1787, Theater nächst der Burg

Leopold Koželuh: Moise in Egitto (Leitung: Koželuh)

Koželuh; Klavierkonzert (M. Th. Paradies)

15. 3. 1788, Wiederholung: 16. 3. 1788, Theater nächst der Burg

Dominicus Mombelli: La morte, e la deposizione dalla croce di Gesu Cristo (Leitung: Salieri)

Johann Hofmann: Concertino

Georg Albrechtsberger: neuer Chor

22. 12. 1788, Wiederholung: 23. 12. 1788, Theater nächst der Burg

Joseph Haydn: 2 Symphonien

Georg Friedrich Händel: 2 Arien mit Chor

Sacchini: Chor

Rondo (gesungen von der Ferraresi)

Händel: Chor

Violinkonzert (Josef Hofmann)

Kontrabasskonzert (Johann Sperger)

Aria (gesungen von Herrn Morella)

Leitung: Salieri

am 23. 12.: Programm wie am 22., jedoch mit neuen Konzerten:

Fortepianokonzert (Frl. Auernhammer)

Violinkonzert (Josef Hofmann)

Violoncellokonzert (Joseph Schindlöcker)

Leitung: Salieri

1. 4. 1789, Wiederholung: 5. 4. 1789, Theater nächst der Burg

Dittersdorf: die besten Nummern aus Giobbe

Albrechtsberger: Chor „Halleluja“

Violoncellokonzert (Max Willmann, 1. 4.)

Violinkonzert (Heinrich Eppinger, 5. 4.)

Leitung: Salieri 


\section{Karwoche 1790: keine Tonkünstler-Konzerte (Landestrauer um Joseph II.)}

22. 12. 1790, Wiederholung: 23. 12. 1790, Theater nächst der Burg

Leopold Koželuh: Moise in Egitto (Leitung: Koželuh)

Koželuh: Konzert für Pianoforte (Marie Therese Paradies, 22. 12.)

Anton Wranitzky: Violinkonzert (A. Wranitzky)

16. 4. 1791, Wiederholung: 17. 4. 1791, Theater nächst der Burg

Mozart: Symphonie

Giovanni Paisiello: Ausschnitte aus der Oper Phädra/Fedra

Mozart: Aria (Mme Lange)

Pleyel: Violoncellokonzert (Cajetan Gottlieb, 16. 4.)

Friedrich Dürand: Violionkonzert (Dürand, 17. 4.)

Albrechtsberger: Chor „Halleluja“

Druschetzky: Musik für 21 Holzblasinstrumente (komponiert für die Krönung in Prag, gespielt von

Mitgliedern der Harmoniemusik der Fürsten Esterhazy und Grassalkovic)

Leitung: Salieri

Kein Tonkünstler-Konzert im Advent [musste wegen der Erkrankung eines Sängers abgesagt werden].

15. 4. 1792, Wiederholung: 16. 4. 1792, Theater nächst der Burg

Joseph Haydn: Symphonie

Oboenkonzert (Josef Triebensee)

Anton Kraft d. Ä.: Violoncellokonzert (A. Kraft d. J., 15. 4.)

Krumpholz: Harfenkonzert (Josefa Müllner, 16. 4.)

Pleyel: Aria (15. 4.)

Borghi: Aria (16. 4.)

Bianchi: Aria (15.4.)

Prati: Aria (16. 4.)

Sacchini: Duett

Albrechtsberger: Quintett in pieno

Händel: Chor

Haydn: Chor

Albrechtsberger: Chor

Leitung: Salieri

22. 12. 1792, Wiederholung: 23. 12. 1792 [wurde wegen der Erkrankung einer Sängerin abgesagt],

Theater nächst der Burg

Joseph Weigl: Venere e Adone (Kantata)

Josef Preindl: Fortepiano-Konzert (Cäsar Scheidl)

Leitung: Josef Weigl

23. 3. 1793, Wiederholung: 24. 3. 1793, Theater nächst der Burg

Joseph Weigl: Venere e Adone (Kantata)

Josef Hofmann: Violinkonzert (Josef Hofmann, 23. 3.)

Josef Preindl: Fortepiano-Konzert (Johanna Sonnleithner, 24. 3.)

Leitung: Josef Weigl

22. 12. 1793, Wiederholung: 23. 12. 1793, Theater nächst der Burg

Joseph Haydn: Symphonie

Aria [keine Nennung des Komponisten] 
Haydn: Chor [mit deutschem Text]

Haydn: Symphonie

Violinkonzert (Heinrich Eppinger, 22. 12.)

Aria (23. 12.)

Went: Trio für 2 Oboen und Englischhorn (Johann, Franz und Philipp Teimer, 23. 12.)

Haydn: Chor [mit italienischem Text]

Haydn: Symphonie

Leitung: Salieri

[alle Haydn-Stücke seien bei den England-Aufenthalten komponiert worden]

12. 4. 1794, Wiederholung: 13. 4. 1794, Theater nächst der Burg

Joseph Haydn: Symphonie (,mit dem Paukenschlag“)

Arie mit obligater Flöte

Joseph Preindl: Chor

Aria

Vicenzo Righini: neues Vokalquartet

Doppelkonzert für Horn (Gabriel Lendway, Matthäus Nickel, 12. 4.)

Violinkonzert (Franz Clement, 13. 4.)

Johann Paisiello: Hymnus (für 2 Orchester und 2 Chöre)

Leitung: Salieri

22. 12. 1794, Wiederholung: 23. 12. 1794, Theater nächst der Burg

Joseph Eybler: Die Hirten bei der Krippe zu Bethlehem

Josef Triebensee: Oboenkonzert (J. Triebensee, 22. 12.)

Violoncellokonzert (Hauschka, mit Variationen über ein Thema aus der Zauberflöte, 23. 12.)

Leitung: Salieri

29. 3. 1795, Wiederholung: 30. 3. 1795, Theater nächst der Burg

Cartellieri: Gioas, re de Giuda (1. Teil: 29. 3., 2. Teil 30. 3.)

Cartellieri: Symphonie

Ludwig von Beethoven: Konzert für Fortepiano (Beethoven, 29. 3.)

Cartellieri: Fagottkonzert (Matuschek, 30. 3.)

Leitung: Salieri

22. 12. 1795, Wiederholung: 23. 12. 1795, Theater nächst der Burg

Paul Wranitzky: Symphonie

Vincenzo Righini: Aria mit Chor (22. 12.)

Salieri: Aria mit Oboe (23. 12.)

Stengel: Aria mit obligater Oboe und Englischhorn

Sarti: Aria (22. 12.)

Zingarelli: Vokalrondo (23. 12.)

Salieri: Vokalrondo

Pugnani: Cavatina (für Gesang und Gitarre)

Paisiello: Vokaltrio

Viotti: Doppelkonzert für Violine (22. 12.)

Schindlöcker: Violoncellokonzert (Joseph Kremer, 23. 12.)

Zingarelli: Quintett für Gesang

Händel: Chor

Sacchini: Chor

Haydn: Chor

Albrechtsberger: Chor (23. 12.)

Leitung: Salieri 
20. 3. 1796, Wiederholung: 21. 3. 1796, Theater nächst der Burg

Salieri: La Riconoscenza (Cantata für Sopran-Solo)

Luigi Tomasini d. Ä.: Violinkonzert (L. Tomasini d. J., 20. 3.)

Harfenkonzert (Josefa Müllner, 21. 3.)

Winter: Timotheus, oder die Gewalt der Musik

Leitung: Salieri

22. 12. 1796, Wiederholung: 23. 12. 1796, Theater nächst der Burg

Paul Wranitzky: neue Symphonie

Franz Xaver Süßmayr: Der Retter in Gefahr ${ }^{28}$ (Kantate)

Haydn: Chor

Haydn: Andante aus der Symphonie mit dem Paukenschlag

Fortepiano-Konzert (Wölfel, 22. 12.)

Violoncellokonzert (Schindlöcker, 23. 12.)

Leitung: Salieri

9. 4. 1797, Wiederholung: 10. 4. 1797, Theater nächst der Burg

Winter: Timotheus, oder die Gewalt der Musik

Triebensee: Konzert für Violine und Oboe (Triebensee, Breymann, 9. 4.)

Cartellieri: Klarinettenkonzert (Brüder Stadler, 10. 4.)

Leitung: Salieri

22. 12. 1797, Wiederholung: 23. 12. 1797, Theater nächst der Burg

Paul Wranitzky: Symphonie

Händel: Chor

Süssmayr: ,, das beliebte Andante“ aus Der Retter in Gefahr

Krammer: Oboenkonzert (Czerwenka, 22. 12.)

Anton Wranitzky: Konzert für Violine und Violoncello (Wranitzky, Kraft, 23. 12.)

Albrechtsberger: „Halleluja“ (Chor)

Cimarosa: Aria

Righini: Aria

Sacchini: Chor (22. 12.)

Beethoven: Trio mit Variationen aus Don Juan für 2 Oboen und Englischhorn (23. 12.)

Righini: Vokalquartett mit Chor

Leitung: Salieri

1. 4. 1798, Wiederholung: 2. 4.1798, Theater nächst der Burg

Eybler: Symphonie

Klarinettenkonzert (Bähr, 1. 4.)

Beethoven: Quintett op. 16 (2.4.)

Joseph Haydn: Die sieben Worte (Vokalfassung)

Leitung: Joseph Haydn

22. 12. 1798, Wiederholung: 23. 12. 1798, Theater nächst der Burg

Eybler: Symphonie

Joseph Haydn: Aria

28 Diese Kantate war am 19. September 1796 bei einer Benefizvorstellung zu Gunsten des Corps der Wiener Freiwilligen im Großen Redoutensaal der Hofburg uraufgeführt worden (mit den Damen Gassmann, Willmann und Tepfer sowie den Herrn Saal und Schulz unter der Leitung von Joseph Scheidl); die Aufführungen durch die Tonkünstler-Societät waren bereits die Wiederholungen vier und fünf dieser offenbar sehr beliebten „Patriotischen Komposition“. 
Haydn: Militär-Symphonie (Leitung: Haydn)

Koželuh: Konzert (für Fortepiano, Mandoline, Trompete und Kontrabass)

Romagnoli: Kantate mit Chor

Leitung: Salieri

17. 3. 1799, Wiederholung: 18. 3. 1799, Theater nächste der Burg

Gluck: Symphonie

Haydn: Die sieben Worte (Vokalfassung)

Triebensee: Oboen-Konzert (Triebensee, 17. 3.)

Peter Fux: Violinkonzert (Fux, 18. 3.)

Leitung: Haydn

22. 12. 1799, Wiederholung: 23. 12. 1799, Theater nächst der Burg Joseph Haydn: Die Schöpfung

Leitung: Haydn

6. 4. 1800, Wiederholung: 7. 4. 1800, Theater nächst der Burg

Haydn: Die Schöpfung

Leitung: Haydn?

22. 12. 1800, Wiederholung: 23. 12. 1800, Theater nächst der Burg Haydn: Die Schöpfung

Leitung: Haydn?

29. 3. 1801, Wiederholung: 30. 3. 1801, Theater nächst der Burg

Eybler: Symphonie

Haydn: Die sieben Worte (Vokalfassung)

Violinkonzert (Luigi Tomasini, 29. 3.)

Schindlöcker: Violoncellokonzert (Schindlöcker, 30. 3.)

Leitung: Haydn

22. 12. 1801, Wiederholung: 23. 12. 1801, Theater nächst der Burg Joseph Haydn: Die Jahreszeiten

Leitung: Haydn

11. 4. 1802, Wiederholung: 12. 4. 1802, Theater nächst der Burg

Joseph Haydn: Die Jahreszeiten

Leitung: Haydn

22. 12. 1802, Wiederholung: 23. 12. 1802, Theater nächst der Burg

Joseph Haydn: Die Jahreszeiten

Leitung: Haydn

3. 4. 1803, Wiederholung: 4. 4. 1803, Theater nächst der Burg

Joseph Haydn: Symphonie

Ferdinand Paer: Das heilige Grab (Oratorium)

Anton Wranitzky/Martin Schlesinger: Doppelkonzert für 2 Violinen (Wranitzky, Schlesinger, 3. 4.)

Witt: Klarinettenkonzert (Joseph Bähr, 4. 4.)

Leitung: Paer

22. 12. 1803, Wiederholung: 23. 12. 1803, Theater nächst der Burg

Abbé Vogler: Kastor und Pollux (Castore e Polluce) 
Leitung: Vogler

25. 3. 1804, Wiederholung: 26. 3. 1804, Theater nächst der Burg Joseph Haydn: Die Schöpfung

Leitung: ?

22. 12. 1804, Wiederholung: 23. 12. 1804, Theater nächst der Burg Joseph Haydn: Die Jahreszeiten

Leitung: ?

7. 4. 1805, Wiederholung: 8. 4. 1805, Theater nächst der Burg Joseph Haydn: Die Schöpfung

Leitung: ?

22. 12. 1805, Wiederholung: 23. 12. 1805, Theater nächst der Burg Cherubini: Grand Overture

Mussini: Aria

Cherubini: Chor mit Soli

Paer: Aria

Cherubini: Chor

Paer: Aria

Cherubini: Chor mit Soli

Clement: neues Violinkonzert

Sarti: Vokal-Trio

Cherubini: Chor

Leitung: Luigi Cherubini

30. 3. 1806, Wiederholung: 31. 3. 1806, Theater nächst der Burg

Kanne: Symphonie

Preindl: Chor

Nasolini: Aria (30. 3.)

Paer: Aria mit Klarinette (31. 3.)

Sarti: Vokal-Trio

Sacchini: Chor

Mozart: Konzert für Pianoforte (Josef Platzer, 30. 3.)

Rode: Violinkonzert (Urbany, 31. 3.)

Salieri: Kantate (Weinmüller, Ehlers)

Leitung: Salieri

22. 12. 1806, Wiederholung: 23. 12. 1806, Theater nächst der Burg Georg Friedrich Händel: Judas Maccabäus [in der Mozart-Fassung] Leitung: Salieri

22. 3. 1807, Wiederholung: 23. 3. 1807, Theater nächst der Burg

Cherubini: Ouverture zur Anacréon

Haydn: Chor

Johann Nepomuk Hummel: Endimione e Diana (Kantate)

Mozart: Pianoforte-Konzert (Sieber, 22. 3.)

Danzi: Violoncello-Konzert (Legrand, 23. 3.)

Händel: Halleluja (aus dem Messias)

Leitung: Hummel 
22. 12. 1807, Wiederholung: 23. 12. 1807, Theater nächst der Burg Joseph Haydn: Die Schöpfung

Leitung: Salieri

10. 4. 1808, Wiederholung: 11. 4. 1808, Theater nächst der Burg Joseph Haydn: Die Jahreszeiten

Leitung: Salieri

22. 12. 1808 und 23. 12. 1808, Theater nächst der Burg

Sigismund Neukomm: Phantasie für Orchester (22. 12.)

Beethoven: Konzert für Pianoforte

Joseph Haydn: Il ritorno di Tobia (Teil 1: 22. 12., Teil 2: 23. 12.)

Leitung: Neukomm

26. 3. 1809, Wiederholung: 27. 3. 1809, Theater nächst der Burg Joseph Haydn: Die Schöpfung

Leitung: Salieri

22. 12. 1809, Wiederholung: 23. 12. 1809, Theater nächst der Burg Joseph Haydn: Die Jahreszeiten

Leitung: Salieri

15. 4. 1810, Wiederholung: 16. 4. 1810, Theater nächst der Burg Joseph Eybler: Die vier letzten Dinge

Leitung: Salieri

22. 12. 1810, Wiederholung: 23. 12. 1810, Theater nächst der Burg Joseph Haydn: Die Schöpfung

Leitung: Salieri

Elisabeth Fritz-Hilscher (elisabeth.fritz@oeaw.ac.at), Institut für kunst- und musikhistorische Forschungen, Abteilung Musikwissenschaft, Österreichische Akademie der Wissenschaften, Wien. 


\section{ABSTRACT \\ FAMOUS ORATORIOS PRODUCED BY THE TONKÜNSTLER-SOCIE- TÄT IN VIENNA - COUNTERPOINT OR FOLLOWING THE TRADI- TION OF ORATORIOS AT THE BAROQUE HAPSBURG COURT?}

After the death of Charles VI. music at the Hapsburg court in Vienna was gradual reduced by Maria Theresa. Also - similar to opera and theater - performances of oratorios were limited and no longer exclusively at the court and court society. Starting 1745 "Fast concerts" were initiated in the "Theater nächst der Burg". With the organization of major oratorio performances by the 1771 founded "Tonkünstler-Societät" ("Musicians-Societät") a further step has been achieved to a modern concert concept: not only the listener group was extended and changed, but also the performance venue from the immediate area of the Imperial Palace in the city, in the Kärntnertor- or Burgtheater laid; and also the commercial aspect should not be excluded, but it was primarily at these academies to increase the pension and provident fund of the Societät.

But there are also parallels to the baroque oratorios productions: This starts with the topics, goes beyond the texts usually written in imitating Pietro Metastasio's librettos (such as Il ritorno di Tobia, GG Boccherini / Haydn) and ends with the choice of days of performance (Lent, Holy Week) and the conspicuous presence of the Emperor and Empress and members of the imperial family. And even continuity is stated by the performers: The "Tonkünstler-Societät" as (essentially) "Pension Institute of the court musician" was naturally anxious to have involved its members in the first place, so the musicians of the court orchestra and the singers of the court theater.

The paper is mainly focused to the issues of continuity and discontinuity in the detachment of the courtly dominated musical life in Vienna by a publicly-commercial as well as the redefinition of the genre oratorio in Vienna 1770-1810.

\section{Key words}

Vienna, oratorio, 18th century, Viennese classic, concert life

\section{Bibliography}

EYBL, Martin - FRITZ-HILSCHER, Elisabeth. Vom Barock zur Wiener Klassik. In Wien Musikgeschichte. Von der Prähistorie bis zur Gegenwart. Eds. Fritz-Hilscher, Elisabeth - Kretschmer, Helmut. Münster-Berlin: Lit 2011, S. 213-269.

GROSSEGGER, Elisabeth. Theater, Feste und Feiern zur Zeit Maria Theresias 1742-1776. Nach den Tagebucheintragungen des Fürsten Johann Joseph Khevenhüller-Metsch, Obersthofmeister der Kaiserin. Wien: Verlag der ÖAW 1987 (Veröffentlichungen des Instituts für Publikumsforschung 12).

HELLSBERG, Clemens. Demokratie der Könige. Die Geschichte der Wiener Philharmoniker. Zürich-Wien-Mainz: Kemayr \& Scheriau 1992.

KUBISKA, Irene. ,Zwischen Anspruch und Gnade“ - Die Supplikationen Wiener Hofbediensteter an den Kaiser in der Mitte des 18. Jahrhunderts. Wien: Masterarbeit 2011.

MORROW, Mary Sue. Concert Life in Haydn's Vienna: Aspects of a Developing Musical and Social Institution. Stuyversant: Pendragon Press 1989 (Sociology of music 7).

Mozart. Briefe und Aufzeichnungen. Gesamtausgabe. Ed. Internationalen Stiftung Mozarteum, gesammelt und erläutert von Deutsch, Otto Erich. Band III: 1780-1786. Kassel-Basel-London: Bärenreiter 1987.

Mozart. Dokumente seines Lebens. Gesammelt und erläutert von Deutsch, Otto Erich. Basel-London-New York: Bärenreiter 1961 (Wolfgang Amadeus Mozart, Neue Ausgabe sämtlicher Werke $\mathrm{X} / 34)$. 
OBROVSKI, Herta. Das Wiener Vereinswesen im Vormärz. Wien: phil. Diss. 1970.

PETE, Claudia. Geschichte der Wiener Tonkünstler-Societät. Wien: phil. Diss. 1996 [ungedruckt].

SCHÖPFER, Gerald. Sozialer Schutz im 16.-18. Jahrhundert. Graz: Leykam 1976 (Grazer Rechtsund Staatswissenschaftliche Studien 33).

SCHÜTZ, Karl. Musikpflege an St. Michael in Wien. Wien: Verlag der ÖAW 1980 (Veröffentlichungen der Kommission für Musikforschung 20).

SEIFERT, Herbert. Barock. In: Wien Musikgeschichte. Von der Prähistorie bis zur Gegenwart. Eds. Fritz-Hilscher, Elisabeth - Kretschmer, Helmut. Münster-Berlin: Lit 2011, S. 143-212. 